\title{
XXIII.
}

\section{Ueber die hämorrhagische Infection.}

\author{
(Vorläufige Mittheilung.) \\ Yon \\ Prof. Dr. Antonio Ceci.
}

(Aus dem pathologisch - anatomischen Institute zu Prag.)

Das zu den Untersucbungen verwendete Blut stammte von einem 19 jährigen Weibe, welches 3 Tage vorher auf die Klinik von Prof. Jacksch mit hohem Fieber, Delirium, Blut und Albumen im Harn, rothen Flecken und dann Hämorrhagien in der Haut gekommen wä. Die klinische Diagnose lautete auf Scarlatina haemorrhagica.

Bei der im pathologisch-anatomischen Institute vom Assistenten Dr. Dreuschuch ausgeführten Section fand man: Punktförmige Hämorrbagien und Phlyctänen in der Hant. Die Meningen hyperämisch und mit Hämatin suffundirt: das Blut überall flüssig und dunkel. Unterhautbindegewebe blutig suffundirt. Petechien im Pericardium und Hämorrhagien im Myocard. Hyperämie der Bronchien. Hämorrhagien in der Pleura. Nekrotische Schorfe an den Tonsillen. Aorta blutig imbibirt. Peritonealflüssigkeit blutig-serös. Extravasationen im Peritoneum. Milz gross und weich. Ekchymosen unter der Nierenkapsel. Reichliche Hämorrhagien in den Nierenbecken und Harnkanälchen. Ekchymosen in der Magenschleimhaut. Hämorrhagien in der Dickdarmschleimhaut, unter der Leberkapsel, in den Ovarien und dem Rectum. Frische Schnitte des Kehlkopfs, sowie einige Stellen des Peritoneum zeigten unter dem Mikroskope in den Capillaren Mikrokokkenanbäufungen, eingebettet in Zooglöa.

Es wurde die anatomische Diagnose auf Variola haemorrhagica gestellt. ${ }^{1}$ ) $\mathrm{Zu}$ der Zeit herrschte in Prag eine bedeutende Blatternepidemie.

1) Ich habe diese Diagnose beanstandet, weil die auch für Variola haemorrh. charakteristische Rachenaffection fehite, und veranlasste deshalb Prof. Ceci, die Natur des jedenfalls neuen Krankheitsprocesses experimentell zu studiren. 
Von dem den Jugular- und Nierenvenen entnommenen Blute wurden einige Tropfen unter den Conjunctivalfornix eines Kaninchens injicirt; mit dem übrigen Theil zwei Culturen angestellt: die eine in Hausenblase, die andere in Urin.

Das Kaninchen ging zwei Tage nach der Einimpfung zu Grunde, obne erhebliche Fiebererseheinungen dargeboten zu haben. Die Seetion ergab überall dunkles, flussiges Blut und Hämorrhagien in der ganzen Musculatur, vorzugsweise aber in der des Nackens, des Ruickens und der Extremitäten. Hämorrhagien in den Hirn- und Ruickenmarkshäuten, in den Lungen und Nieren. Unter dem Mikroskope fand man kugelige, stark lichtbrechende, bewegliche Körperchen von äusserster Kleinbeit in der Milzpulpa, im Knochenmark des Femur und, weniger zahlreich, im Blute. Bei der mikroskopischen Untersuchung sei es von tingirten Schnitten der frischen Organe, sei es von dünnen Membranen, fand man an den Blutgefässen nichts Abnormes. Die morphologischen Elemente des Blutes waren normal. Das Blut dieses Kaninchens wurde einem zweiten eingeimpft, das des zweiten einem dritten, das des dritten einem vierten, das des vierten einem füften, das des fünften einem sechsten. Das zweite, dritte und fünfte Kaninchen erlagen nacheinander einige Tage nach der Inoculation; das vierte und sechste wurden getödtet. Das dritte, vierte und fünfte boten diffuse Hämorrhagien dar; beim zweiten und sechsten wurden keine vorgefunden.

Die zwei Kaninchen eingeimpfte Hausenblasencultur des menschlichen Blutes hatte weder Hämorrhagien noch den Tod zur Folge.

Die Harncultur des menscblichen Blutes tödtete dagegen ein Kaninchen vier Tage, ein zweites 27 Stunden und ein drittes zwei Tage nach der Einimpfung. Alle zeigten bei der Section zahlreiche Hämorrhagien. Mit dem Blute des dritten Kaninchens wurden zwei Parallelculturen angestellt: die eine in Urin, die andere in Hausenblase. Die einem Kaninchen beigebrachte Harncultur tödtete dasselbe durch Hämorrhagien, während die Hausenblasencultur bei einem anderen Kaninchen ohne Folgen blieb.

Von demselben dritten Kaninchen erhielt man durch successive Bluteinimpfungen eine Reibe von vier Kaninchen, die nacheinander an Hämorrhagien zu Grunde gingen. Das Blut des ersten Kaninchens dieser Reihe tödtete durch Hämorrhagien zwei Frösche, die man damit geimpft batte; während es bei zwei Hunden und zwei Tauben ohne alle Wirkung blieb.

Mit dem Blute dieses selben Kaninchens, des ersten von der Reihe wurden zwei Harneulturen angestellt, von denen man die eine 
unmittelbar nach ihrer Anfertigung kochte. Die gekochte Cultur äusserte nach ihrer Einimpfung keine Wirkungen; die andere tödtete dagegen ein Kaninchen durch Hämorrhagien.

Die mit dem menschlichen Blute angestellte, mehrere Tage lang in offenem Gefässe stehen gebliebene und der Fäulniss verfallene Harneultur blieb, einem Kaninchen eingespritzt, wirkungslos. Eine weitere, aus dieser Cultur gewonnene und einem Kaninchen eingeimpfte Harncultur ergab kein Resultat. Ebenso unwirksam erwies sich eine Cultur des menschlichen Blutes, die nach vorherigem Kochen in reichlicher Menge in die Peritonealhöhle eines anderen Kaninchens eingespritzt wurde.

Beztiglich der Culturen ergab sich: dass weder das Blut der menschlichen Leiche, noch dasjenige der an Hämorrhagien zu Grunde gegangenen Kaninchen, in Hausenblase cultivirt, irgend welche Wirkungen äusserten.

Für gewöhnlich erhielt sich die Culturflüssigkeit klar, an der Oberfläche rein und bildete keinen Bodensatz. Kein Fäulnissgeruch; die Reaction blieb auf unbestimmte Zeit entweder sauer oder neutral.

Unter dem Mikroskope zeigte sie meistens keine Organismen. Ungefähr das nämliche Verhalten ergab sich auch in Bezug auf Hausenblaseneulturen, zu denen man einige Tropfen einer Harncultur als inficirenden Stoff angewendet hatte.

Sowohl das menschliche als auch das Blut von an Hämorrhagien zu Grunde gegangenen Kaninchen gab stets positive Resultate. Die Flïssigkeit trübte sich gewöhnlich einige Zeit nach der Infection: sie entwickelte Fäulnissgeruch und nahm alkalische Reaction an. In einem Falle blieb letztere neutral, und es trat keine Fäulniss ein, obwohl die Cultur sich bei den Versuchsthieren als wirksam erwies.

Die zweiten und dritten successiven Harnculturen des menschlichen Blutes waren' minder üppig: die Trtibung der Flitssigkeit eine geringe; die Fäulniss blieb aus, und die Reaction erhielt sich sauer oder neutral.

Liess man die mit dem Blute von an Hämorrhagien zu Grunde gegangenen Thieren angestellten Culturen wenige Minuten lang aufkochen, so wurde jede Entwicklung von Organismen in denselben hintangehalten.

Die Flusssigkeit solcher nach ihrer Infection gekochten Culturen blieb beständig klar: kein Fäulnissgeruch: Reaction sauer oder neutral: Abwesenheit von Organismen. In sämmtlichen Culturen, deren Anwendung pathologische Folgen nach sich zog, fand man (ent- 
weder allein, oder mit anderen gemischt) zahlreiche Organismen von äusserster Kleinheit und sphärischer oder leicht ovoider Gestalt; manchmal nach Art von kurzen Bacillen verlängert, entweder isolirt oder zu Haufen traubenartig aneinander gruppirt. Oft zeigten diese Organismen lebhafte Bewegungen.

Die Ergebnisse der Versuche an Thieren und der Culturen veranlassen uns zu folgenden Schlissen:

1. Die anatomische Diagnose an der menschlichen Leiche, von der das Blut genommen wurde, war eine unbestimmte.

2. Es gibt eine besondere acute Infection, die sich anatomisch durch diffuse und schwere, die Substanz der Muskeln, die serösen Häute, die Niere, das Knochenmark befallende Hämorrhagien charakterisirt, und durch keinen bestimmten Fiebertypus gekennzeichnet ist. Die geformten Blutbestandtheile scheinen dabei keine Veränderung zu erleiden.

3. Eine derartige Infection kann auf ein gesundes Thier durch directe Einimpfung des Blutes eines von derselben befallenen Thieres tibertragen werden und äussert sich nach einer kurzen, jedoch veränderlichen Zeitperiode.

4. Die Infection kann auch durch Einimpfung von mit dem Blute eines mit demselben Uebel behafteten Thieres angestellten Harnculturen herbeigeführt werden. Die Hausenblasenculturen sind unwirksam.

5. Die Culturen, deren Anwendung positive pathologische Resultate zur Folge hatte, zeigten eine Triibung der Flüssigkeit, häufig Fäulnissgeruch und Alkalescenz. Die beiden letzteren Charaktere waren nicht constant. In morphologischer Beziehung kamen in sämmtlichen Culturen kugelige, ovoide, oder Bacillus-förmige, sich bewegende Organismen von äusserster Kleinheit entweder allein, oder mit anderen gemischt vor.

6. Längeres Stehenbleiben, die Einwirkung der Luft, das längere Aufeinanderfolgen von Generationen machten die Culturen zu pathologischen Wirkungen unfähig.

7. Sowohl im Blute als auch in den Organen der den Hämorrhagien erlegenen Thiere wurden häufig sehr kleine, sich bewegende, das Licht brechende, den in den Culturen beobachteten gleichende Körperchen vorgefunden : niemals aber Verstopfungen von Capillaren durch Anhäufungen solcher Organismen wahrgenommen, deren Feststellung durch ihre Kleinheit sehr erschwert wird.

8. Die beschriebene Infection kann nur an der Hand der parasitären Theorie gedentet werden; nichtsdestowoniger ist die Entstehungsweise von so ausgedehnten und zahlreichen Hämorrhagien schwer zu erklären. Als die wahrscheinlichste Hypothese kann man eine materielle Veränderung der Blutgefässwandungen annehmen, die entweder direct durch locale Verletzung von Seite der vorhandenen Organismen oder aber durch von denselben Organismen bedingte chemische Veränderungen des Blutes zu Stande kommt. Den Muskelbewegungen würde die Rolle von Gelegenheitsursachen zufallen, welche die. Vorliebe solcher Hämorrhagien für die Muskelsubstanz erklären wïrden. 
9. Vorhergegangene Infectionen verliehen den Thieren keine Immunität gegen die hämorrhagische.

10. Die bei den successive geimpften Thieren erzielten Infectionen blieben nach einer gewissen Zeit aus, da die inficirende Flïssigkeit immer mehr an Wirksamkeit einbüsste.

Es ist schwer, eine genaue Bezeichnung für die beschriebene Infection zu wählen, ohne sich dabei durch vorgefasste Meinungen beeinflussen zu lassen. Die Unsicherheit der ätiologischen Diagnose in dem Falle, welcher das Material für die weiteren Versuche lieferte, erhöht diese Schwierigkeit. Den Bezeichnungen hämorrhagisehe Diathese, acute Hämophilie habe ich die einer hämorrhagischen Infection vorgezogen, obwohl auch eine solche keineswegs hinreichend klar und genau ist.

Camerino, 10. Februar 1881.

\section{XXIV. \\ Literarischer Anzeiger.}

No. III (Pathologie).

Der zehnte Congress der deutschen Gesellschaft für $\mathrm{Ch}$ irurgie, welcher soeben unter zahlreicher Betheiligung in Berlin abgehalten ist, brachte auch Einiges in unser Gebiet einschlagendes, dem hier einige Worte gewidmet werden sollen.

Zuerst theilte Grawitz (Berlin) seine weiteren Erfahrungen über die im Körper der Warmblüter sich entwickelnden Fadenpilze mit und zwar in der Absicht, die an diesem Objecte gewonnenen Anschauungen für die Theorie der Vaccination zu verwerthen. Im Allgemeinen ergeben sich für diese Organismen dieselben Verhältnisse wie für die Spaltpilze: Kürzere Zitichtung (in Peptonlösung) oder geringere Menge des Impfmaterials bewirkt leichtere Erkrankung des Versuchsthieres und Schutz vor einer weiteren, auch mit wirksamerem Material ausgeführten Impfung. Abweichend von jenen Erfahrungen an Schistomyceten ist dagegen die Angabe, dass jeder dieser Pilze (Penicillium, Aspergillus und Oidium), prophylaktisch ibertragen, die Entwicklung einer jeden der beiden anderen Formen verhuttet.

Gewiss mit Recht betont Grawitz die Vortheile, welche das leichter zu controlirende Impfmaterial, welches er verwendete, für die Lösung der Frage von der Schutzimpfung im Allgemeinen darbietet; jedoch wird die endgiltige Erklärung dieses wunderbaren Vorganges erst dann zu erwarten sein, wenn wir die chemischen Veränderungen kennen gelernt haben, welche der Organismus des Impfthieres erfährt. Da der Schutz, welcher durch die Impfung gewonnen werden kann, eine gewisse Dauer besitzt, müssen auch die ihm zu Grunde liegenden Veränderungen die gleiche Dauer besitzen und können nicht in der vorübergehenden Zerstörung irgend eines 